\section{CLINICAL REPORT}

\section{Letourneau-Guillon}

T. Krings

\title{
Simultaneous Arteriovenous Shunting and Venous Congestion Identification in Dural Arteriovenous Fistulas Using Susceptibility-Weighted Imaging: Initial Experience
}

SUMMARY: In this short report, we describe the potential contribution of SWI in the noninvasive evaluation of DAVFs. SWI images were compared with DSA for the identification of the location of the fistulous point, the presence of CVR, and the presence of the PPP. In 5 of 6 patients, it was possible to identify the fistulous locations depicted as hyperintensity within venous structures. Cortical venous reflux was underestimated on SWI in 3 cases of robust CVR and not identified in 2 cases of less severe CVR. The PPP seen on angiograms correlated anatomically with increased number, caliber, and tortuosity of hypointense veins seen on SWI. Furthermore, SWI was superior to conventional MR imaging in the detection of these dilated veins. These preliminary results suggest an important role for SWI in the detection and assessment of the complex hemodynamics associated with DAVFs.

ABBREVIATIONS: $A P=$ anteroposterior; $C V R=$ cortical venous reflux; DAVF $=$ dural arteriovenous fistula; $\mathrm{mIP}=$ minimum intensity projection; $\mathrm{MIP}=$ maximum intensity projection; $\mathrm{PPP}=$ pseudophlebitic pattern

$\mathbf{T}$ he clinical and neuroradiologic diagnosis of DAVFs is often challenging. A wide spectrum of clinical presentation has been described. ${ }^{1,2}$ Similarly, the imaging findings are often nonspecific and may mimic a variety of conditions. Most aggressive clinical presentations are either related to venous congestion or rupture of arterialized veins. ${ }^{3,4}$ Venous congestion is caused by increased venous pressure secondary to venous arterialization, sometimes combined to decrease venous outflow from associated venoclusive disorders. Venous congestion, revealed by the PPP on DSA, is generally associated with CVR, though the absence of CVR does not preclude the presence of venous congestion and neurologic complications. ${ }^{3-5}$ Historically, the characterization of DAVF has mainly relied on the presence of CVR, which serves as the basis for the most commonly used DAVF classifications. ${ }^{4,6}$ The integration of venous congestion in DAVF characterization has received less attention despite being clinically relevant. ${ }^{3}$

The advent of SWI provides a new way to assess the complex pathophysiology of DAVFs. Recent case reports and small series highlighted the prominent hypointense vessels seen in DAVFs and the association of this finding with CVR..$^{7-9} \mathrm{~A}$ recent report also highlighted the ability of SWI to identify arteriovenous shunting in brain vascular malformations, revealed by hyperintensity within venous structures. ${ }^{10}$ This hyperintensity has been attributed to a combination of time-of-flight phenomenon intrinsic to high-velocity arterial flow as well as to the lack of paramagnetic phase shift secondary to the

Received April 15, 2011; accepted after revision June 6.

From the Division of Neuroradiology, Department of Medical Imaging, University of Toronto, Toronto Western Hospital, Toronto, Ontario, Canada.

Please address correspondence to Laurent Letourneau-Guillon, MD, Department of Diagnostic Imaging, University Health Network, University of Toronto, 555 University Ave, Toronto, 0N M5G 1X8, Canada; e-mail: laurent_letg@hotmail.com

Indicates open access to non-subscribers at www.ajnr.org

http://dx.doi.org/10.3174/ajnr.A2777 diamagnetic oxyhemoglobin content of arterial blood. ${ }^{10,11}$ If one takes into account these 2 separate observations, it appears that SWI is potentially able to depict both the venous congestion and the arteriovenous shunt surgery in DAVFs, thereby providing a unique noninvasive assessment of hemodynamic alterations associated with this pathology. Our aim was to retrospectively evaluate the use of SWI in DAVF characterization.

\section{Case Series}

\section{Patient Selection}

Our institutional review board approved this study. Between September 1997 and February 2011, 159 patients underwent MR imaging followed by DSA for the diagnosis of DAVFs. From this database, we identified 6 consecutive patients, imaged between December 2008 and November 2010, whose MR imaging studies included SWI. Medical records were reviewed for patient age, sex, clinical presentation, and time interval between MR imaging and DSA.

\section{Imaging Acquisition}

All preoperative MR images were acquired by using a 1.5T MR imaging unit (Magnetom Avanto; Siemens, Erlangen, Germany) with an 8-channel head coil. All studies included routine T1-, T2-weighted, DWI, and FLAIR images. Three patients also received $0.1 \mathrm{mmol} / \mathrm{kg}$ IV of gadobutrol (Gadovist; Bayer Schering Pharma, Berlin, Germany) followed by T1-weighted and MR venographic acquisitions. All SWI sequences were performed prior to gadolinium administration.

Details of the SWI sequence have been described elsewhere. ${ }^{12}$ The detailed imaging parameters used were as follows: TR, $49 \mathrm{~ms}$; TE, 40 $\mathrm{ms}$; number of averages, 1 ; flip angle, $15^{\circ}$; section thickness, $2.6 \mathrm{~mm}$; matrix size, $256 \times 177$; pixel bandwidth, $80 \mathrm{~Hz}$. A rectangular FOV (5/8) of $256 \mathrm{~mm}$ was used. The raw data processing was performed automatically by the Magnetom Vision software (Siemens) and yielded processed magnitude SWI, referred to as "SWI images" throughout the article. mIPs of $20.8 \mathrm{~mm}$ thickness were automatically processed in the axial plane by using the SWI images. In selected cases, 


\begin{tabular}{|c|c|c|c|c|c|c|c|c|c|}
\hline \multicolumn{10}{|c|}{ Angiographic, SWI, and conventional imaging data } \\
\hline \multirow[b]{2}{*}{$\begin{array}{l}\text { Patient No., } \\
\text { Age }(y r) / S e x\end{array}$} & \multicolumn{5}{|c|}{ DSA } & \multicolumn{3}{|c|}{ SWI } & \multirow{2}{*}{$\begin{array}{c}\text { Conventional } \\
\text { MR Imaging } \\
\text { Dilated } \\
\text { Leptomeningea } \\
\text { or Medullary } \\
\text { Vessels }\end{array}$} \\
\hline & $\begin{array}{c}\text { Borden } \\
\text { Classification }\end{array}$ & Fistulous Point & CVR & PPP & $\begin{array}{l}\text { Veno- } \\
\text { Occlusive } \\
\text { Disease }\end{array}$ & $\begin{array}{c}\text { Fistulous } \\
\text { Point } \\
\text { Identification }\end{array}$ & CVR & PPP & \\
\hline 1) $46 / F$ & $\|$ & $\begin{array}{l}\text { Right transverse } \\
\text { sinus }\end{array}$ & Yes & Moderate & Yes & Yes & Less than DSA & $\begin{array}{c}\text { Moderate, similar } \\
\text { extent to DSA }\end{array}$ & Yes \\
\hline 2) $86 / \mathrm{M}$ & $\begin{array}{l}\text { II (retrograde } \\
\text { sinosal } \\
\text { drainage)/III }\end{array}$ & $\begin{array}{l}\text { Multihole fistula } \\
\text { (superior sagittal } \\
\text { sinus, cortical } \\
\text { veins) }\end{array}$ & Yes & Severe & Yes & Yes, both & Less than DSA & $\begin{array}{l}\text { Severe, similar } \\
\text { extent to DSA }\end{array}$ & Yes \\
\hline 3) $70 / \mathrm{M}$ & ॥ & $\begin{array}{l}\text { Left transverse } \\
\text { sinus }\end{array}$ & Yes & Mild & Yes & Yes & CVR not detected & $\begin{array}{l}\text { Mild, similar extent } \\
\text { to DSA }\end{array}$ & No \\
\hline 4) $31 / \mathrm{F}$ & $\|$ & $\begin{array}{l}\text { Left transverse } \\
\text { sinus }\end{array}$ & Yes & Mild & No & Yes & CVR not detected & $\begin{array}{l}\text { Mild, similar extent } \\
\text { to DSA }\end{array}$ & No \\
\hline 5) $37 / F$ & $\begin{array}{l}\text { Il (retrograde } \\
\text { sinosal } \\
\text { drainage)/ll }\end{array}$ & $\begin{array}{l}\text { Multihole fistula } \\
\text { (right transverse } \\
\text { sinus, superior } \\
\text { sagittal sinus) }\end{array}$ & Yes & Severe & Yes & Yes, both & Less than DSA & $\begin{array}{l}\text { Severe, similar } \\
\text { extent to DSA }\end{array}$ & Yes \\
\hline 6) $64 / \mathrm{M}$ & I & Clivus & No & No & No & No & No & No & No \\
\hline
\end{tabular}

MIP reconstructions of varying thickness were also postprocessed by using the SWI images.

Diagnostic intra-arterial cerebral angiograms were obtained on a dedicated biplane neuroangiographic unit (Infinix; Toshiba Medical Systems, Tochigi, Japan). It generally consisted of bilateral injections of the ICA, external carotid artery, and vertebral artery, supplemented with selective injections of the occipital, ascending pharyngeal, or internal maxillary arteries, when indicated.

\section{Image Analysis}

SWI, other conventional MR imaging sequences, and DSA images were reviewed independently in 3 different sessions by using anonymized images. The images were reviewed by the 2 authors who were blinded to patients' clinical and conventional imaging findings or angiographic data. The reviewers were not blinded to the diagnosis of DAVF but were not aware of the fistula location or grade. The presence of a PPP on SWI was defined by an increased number, increased tortuosity, and increased caliber of leptomeningeal and/or medullary veins compared with normal-appearing regions of the brain. We further graded the severity of the PPP by using a semiquantitative scale depending on the number of dilated veins seen in a particular region: mild PPP, $<5$; moderate PPP, 5-10; and severe PPP, $>10$ veins being larger than those in normal-appearing regions of the brain. The SWI images were also evaluated for the "fistulous point," which was defined as hyperintensity within at least 1 venous structure (ie, the cortical vein or venous sinus). This criterion has been validated in a recent study of brain vascular malformation associated with arteriovenous shunt surgery. ${ }^{10}$ We used the proximal intracranial arteries at the circle of Willis (supraclinoid ICAs, M1 segments, basilar trunk) as internal standards; the signal intensity within the veins or dural sinuses needed to be equal to or greater than these arteries.

Similarly, the "presence of CVR" on SWI was defined as hyperintensity in cortical venous structures radiating from the presumed fistulous point. The presence of other intracranial abnormalities on SWI was also recorded. Disagreements were resolved by consensus. The T1- and T2-weighted, FLAIR, DWI, and T1 postgadolinium sequences (if available), herein labeled conventional imaging sequences, were reviewed for leptomeningeal and/or medullary vascu- lar dilation and/or enhancement as described by Kwon et al. ${ }^{13}$ The DSA images were reviewed in a subsequent session. The DAVFs were classified according to Borden et al. ${ }^{6}$ Additional information recorded on DSA images included location of the fistulous point, presence and location of CVR, and presence and location of a PPP. To ensure that CVR and the PPP were correctly localized on DSA, we reviewed both projections (ie, AP and lateral) before correlating them with the anatomic site of SWI vascular abnormalities. The severity of the PPP was further graded on a 3-point scale (mild, moderate, severe) by using the classification proposed by Willinsky et al. ${ }^{3}$ The presence of venoclusive disease in the major venous sinuses (ie, occlusion or narrowing) was also noted, by using the venous phase of DSA, and was confirmed on a MR venography sequence if available.

\section{Results}

The main findings are summarized in the Table. The main imaging findings in patient 2 (Fig 1), patient 3 (Fig 2), and patient 5 (Fig 3 ) are illustrated below. Clinical presentation included isolated tinnitus $(n=4)$, focal neurologic deficit $(n=1)$, and alteration of level of consciousness $(n=1)$. The mean time interval between MR imaging and DSA was 27 days (range, 3-77 days). In 5 of 6 patients, SWI could demonstrate the location of the fistulous point revealed by hyperintensity within the venous structures. In 1 patient harboring a small clival DAVF without CVR (patient 6), susceptibility artifacts at the skull base impaired identification of the fistula site on SWI. In 2 instances of retrograde filling of dural sinuses, SWI demonstrated high signal intensity in the corresponding venous sinuses (patient 2, Fig $1 E$; patient 5 , Fig $3 D,-E$ ). Among 5 patients with CVR on DSA, SWI hyperintensity in corresponding cortical veins was identified in 3 (patient 2, Fig $1 F$ ). The extent of CVR was significantly less than that observed on DSA. In 2 instances demonstrating mild-to-minimal CVR on DSA, this feature could not be identified on SWI (patients 3 and 4 , respectively).

In all 5 patients demonstrating CVR, variable severity of the PPP was detected on DSA. The severity and extent of the PPP was similar between SWI and DSA. In all instances, SWI 

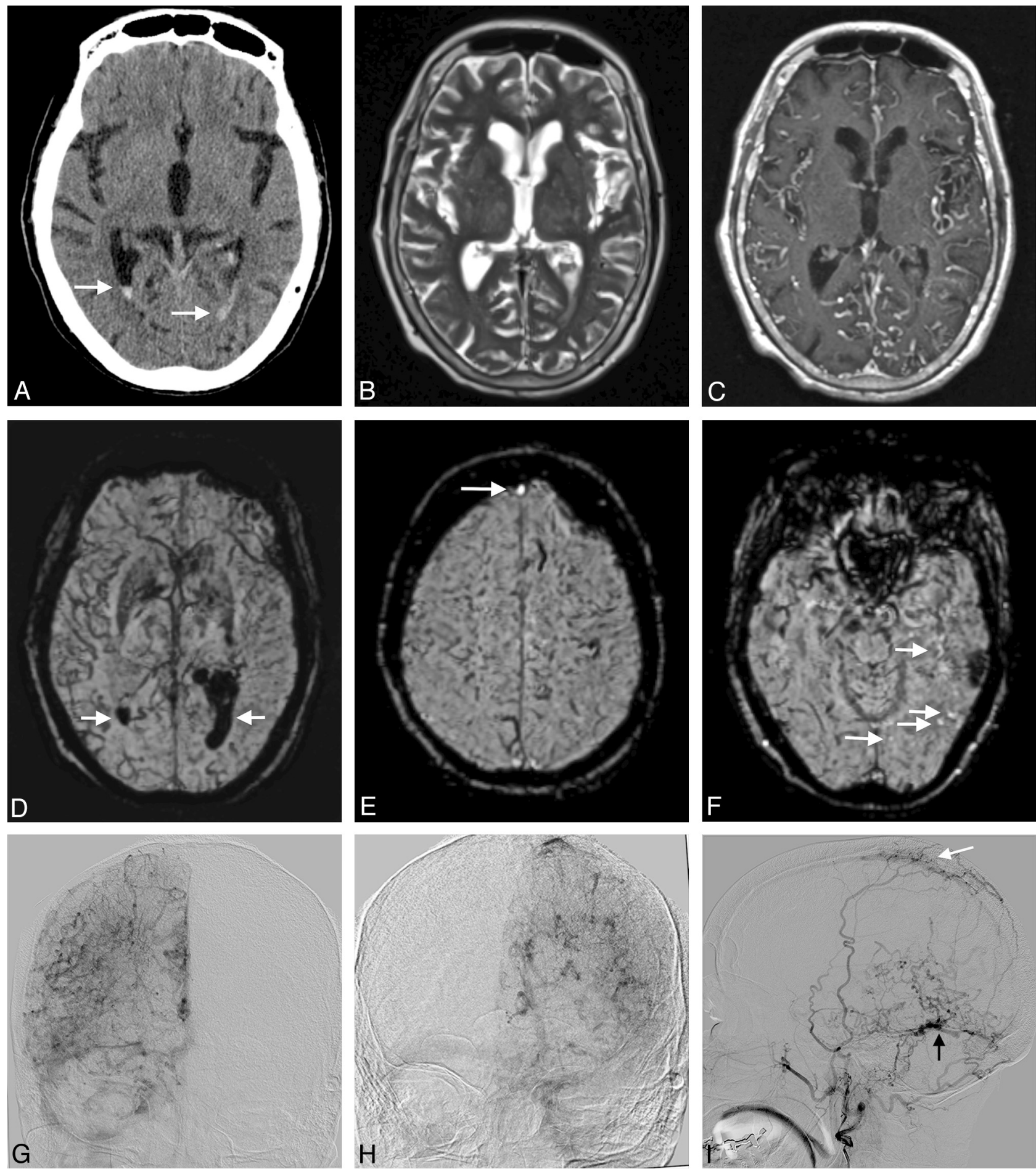

Fig 1. Multifocal DAVF (Borden type II and III) in an 86-year-old man presenting with aphasia (patient 2). A, Unenhanced CT scan demonstrates acute intraventricular hemorrhage (arrows). $B$, T2-weighted image shows a few slightly prominent leptomeningeal vessels. $C$, T1-weighted postgadolinium image better demonstrates the increased number of tortuous leptomeningeal vessels within both hemispheres. There are also a few tortuous medullary vessels traversing the brain parenchyma. D, mIP obtained from SWI images reveals markedly increased leptomeningeal and medullary hypointense veins affecting more severely the right hemisphere (severe PPP). Intraventricular hemorrhage is also identified (arrows). E and $F$, SWI images depict the hyperintensity in the anterior aspect of the superior sagittal sinus, corresponding to retrograde drainage on DSA (arrow in $E$ ). A few hyperintense veins corresponding to CVR in the vicinity of this Borden III fistula (not shown) are also identified (arrows in $\mathrm{A}$. G and H, AP projections of the right and left ICA angiograms obtained in the venous phase. A severe PPP is seen bilaterally, worse on the right side, similar to the findings on SWI. The left transverse and sigmoid sinuses are occluded. I, Lateral projection of a left external carotid artery angiogram in the late arterial phase. Two DAVFs are identified, 1 located in the wall of the occluded left transverse sinus (black arrow) draining directly in cortical veins and another draining into the superior sagittal sinus (white arrow). Retrograde filling of the anterior part of the superior sagittal sinus is present, while the posterior part of the superior sagittal sinus was occluded (not shown). The extent of CVR is more extensive on DSA than depicted on SWI.

showed significantly more dilated hypointense veins than either T2-weighted or T1 postgadolinium sequences. In fact, it is the presence of this finding in those 5 previously undiagnosed cases that initially increased the suspicion of DAVF and subsequently lead to DSA. Furthermore, in 2 cases of mild PPP on DSA (patients 3 and 4), an increased number of tortuous veins 
was seen only on SWI but not on other MR images. Finally, in 1 patient who presented with acute intraventricular hemorrhage, SWI demonstrated marked intraventricular hypointensity (patient 2, Fig 1D). In the same patient, mild pial siderosis was also detected within the posterior fossa, a finding not revealed on T2-weighted imaging (not shown).

\section{Discussion}

In 5 of 6 patients, it was possible to correctly identify the location of the fistula shown as hyperintense venous structures on SWI. This preliminary result is similar to the high sensitivity of 93\% obtained in a recent study including 29 brain vascular malformations (5 DAVFs) associated with arteriovenous shunting. ${ }^{10}$ Tsui et $\mathrm{al}^{11}$ also described hyperintensity in a venous varix related to a DAVF. The hyperintense venous sinuses or veins were well depicted in all cases by the use of SWI images. MIPs obtained from the same SWI images enhanced the visualization of venous hyperintensity (Fig $2 D$ and Fig $3 E$ ), though this was not necessary for the diagnosis. As expected, the use of mIP negated this finding in most cases. We postulated that CVR would manifest with cortical venous hyperintensity radiating from the fistulous point. This assumption was based on the principle that veins affected by CVR contain rapidly flowing and nonparamagnetic arterial blood. The extent of CVR revealed on SWI was, however, underestimated in 3 cases of robust CVR, whereas it could not be identified in 2 instances of scant CVR. Slow-flowing DAVFs may result in poor flow-related enhancement, which may at least partially explain the lack of venous hyperintensity in these 2 falsely negative cases. Furthermore, oxyhemoglobin contained in CVR might be rapidly diluted in deoxyhemoglobin-rich congested veins.

Given this relative underestimation of CVR on SWI, this preliminary observation suggests that the noninvasive evaluation of CVR should rely on time-resolved MRA or CTA, which have sensitivity $>90 \%$ with good-to-excellent interobserver agreement. $^{14-19}$ It is, nonetheless, possible that slow-flowing CVR or fistulas may be missed by using these techniques, given the lack of selective arterial injection and lesser temporal resolution compared with DSA. This situation has recently been encountered in a series of DAVFs evaluated by dynamic CTA ${ }^{14}$ and highlights the persisting role of conventional angiography in DAVF characterization.

Prominent dilated leptomeningeal and medullary veins on SWI were characteristic features of all cases associated with both the PPP and cortical venous drainage. SWI provided excellent depiction of these collateral venous networks, demonstrating more congested veins compared with conventional sequences, a finding enhanced by the use of mIPs. This finding is similar to previous reports on the use of SWI for the depiction of venous congestion secondary to DAVFs. ${ }^{7-9}$ Saini et $\mathrm{al}^{7}$ also described reduction in the prominence of these cortical veins after treatment in 1 patient. A similar observation has been described in the setting of cortical venous outflow obstruction seen in Sturge-Weber syndrome, in which SWI was more sensitive than contrast-enhanced T1-weighted imaging in the detection of transmedullary veins. ${ }^{20}$

In our opinion, the dilated hypointense veins identified on SWI in the setting of DAVFs likely represent enlarged and tortuous collateral veins serving to reroute venous blood away from obstructed and/or hypertensive venous drainage path- ways. ${ }^{3}$ The underlying mechanism for increased venous visibility on SWI is probably multifactorial. It has been postulated that venous hypertension secondary to DAVF leads to hypoperfusion and possibly increased oxygen extraction in ischemic tissue, which would then lead to increased deoxyhemoglobin concentration and increased susceptibility effects. ${ }^{7}$ With positron-emission tomography studies, increased oxygen extraction fraction has been demonstrated in a subset of DAVFs associated with decreased cerebral blood flow and venous-drainage impairment when oxygen metabolism was preserved. ${ }^{21,22}$ In addition to increased oxygen extraction fraction, the increased volume of deoxyhemoglobin within dilated veins may also explain their conspicuity on SWI.

In rare situations, venous congestion may be seen without accompanying CVR. ${ }^{3-5}$ In these instances, there is still the possibility of hemorrhagic or nonhemorrhagic neurologic complications. ${ }^{3-5}$ This scenario is usually associated with arteriovenous shunting within dural sinuses producing increased venous pressure and functional outflow obstruction. In this circumstance, SWI would, in theory, be able to demonstrate engorged veins as well as the hyperintense fistulous point, while the fistula would be classified as benign on the basis of the absence of CVR. The possibility of an independent prognostic value of venous congestion identified on SWI needs further assessment in larger longitudinal studies.

SWI can demonstrate hemorrhagic complications of DAVFs, including previous hemorrhagic events such as pial siderosis or chronic intraparenchymal hematoma that may remain occult on other imaging modalities or MR sequences. Subcortical calcifications in DAVFs ${ }^{23,24}$ could also be identified on SWI by the use of filtered-phase images. ${ }^{25,26}$ Among the disadvantages of SWI is its vulnerability to susceptibility artifacts present at the skull base, as seen in 1 of our cases. Another caveat for SWI in the diagnosis of DAVF is the possibility of fistula obscuration by blood-degradation products as seen in 1 instance in the report from Noguchi et al. ${ }^{8}$ However, in the series from Jagadeesan et $\mathrm{al},{ }^{10}$ the depiction of arteriovenous shunt surgery was paradoxically higher in cases of intracerebral hematoma compared with cases without hemorrhage.

The presence of prominent hypointense pial veins on SWI can also be seen in other conditions, though the presence of marked venous tortuosity would be more suggestive of longstanding venous hypertension as encountered in DAVFs. Furthermore, the additional finding of venous hyperintensity at the fistulous point should also suggest the possibility of a DAVF. Potential mimickers include states of increased oxygen extraction in the setting of oligemia, either in acute ischemic stroke or hemodynamically significant arterial stenosis. ${ }^{25,27}$ Transient unilateral venous prominence has also been described in migraines. ${ }^{28,29}$ Venous congestion secondary to venous thrombosis can also lead to dilated collateral veins, $25,26,30$ but contrary to arterialized veins in DAVFs, the thrombosed veins should appear hypointense from susceptibility effects. ${ }^{31}$ This latter pattern should prompt further evaluation with MR venography because the distinction between patent and thrombosed veins could be difficult on SWI.

The main limitations of this series are the small sample number, retrospective nature, absence of a control arm, and the fact that the readers were aware of the diagnosis of DAVF. 

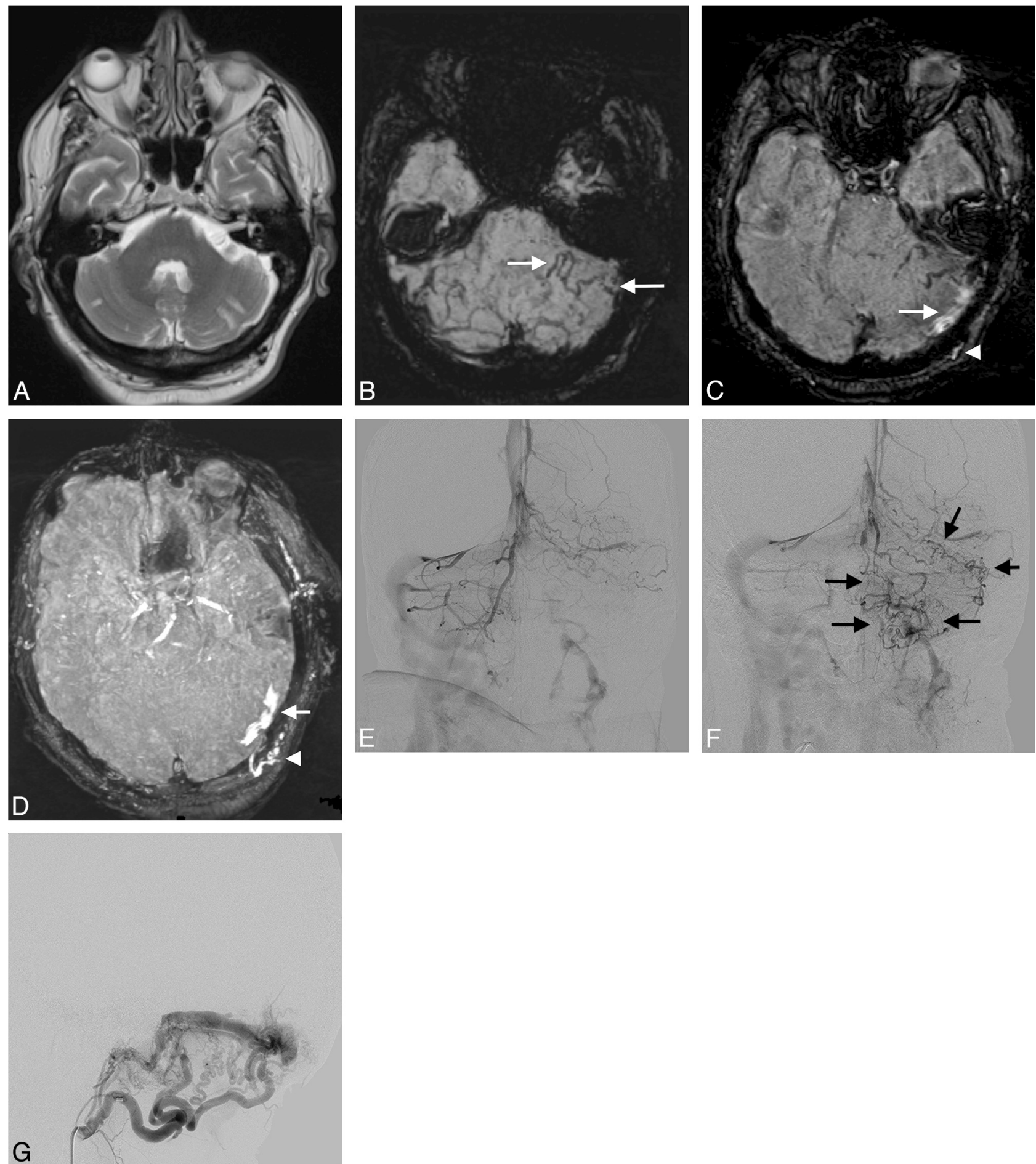

Fig 2. Borden type II DAVF involving the left transverse sinus in a 70-year-old man with extrapyramidal symptoms and progressive right-arm weakness (patient 3). $A$, T2-weighted sequence does not show abnormal vascular structures in the posterior fossa. Chronic infarcts are seen incidentally in both cerebellar hemispheres. $B$, SWI displayed in an mIP reveals enlarged medullary vessels in the left cerebellar hemisphere (mild PPP, arrows). C, SWI demonstrates hyperintensity in the left transverse sinus (arrow) and an enlarged left occipital artery (arrowhead). D, SWI displayed in MIP enhances the depiction of the enlarged right occipital artery (arrow) and hyperintensity in the left transverse sinus (arrowhead). The normal proximal intracranial arteries are also depicted as hyperintense. $E$ and F, AP projections of the right and left vertebral artery angiograms obtained in the venous phase. There is a mild PPP in the left cerebellar hemisphere (arrows), which correlates with the hypointense veins seen on SWI in B. G, Lateral projection of a selective left occipital angiogram obtained in the arterial phase. Marked hypertrophy of the left occipital artery and numerous arterial feeders supply the double-channeled transverse sinus. There was only minimal CVR despite the relatively prominent venous stasis in the left cerebellar hemisphere as revealed on both SWI and the venous phase of the left vertebral artery injection.

In our institution, all dedicated DAVF studies, including posttreatment follow-up MR imaging, include an optimized timeresolved MRA, which is performed on an MR imaging unit without SWI capability, hence the small number of SWI stud- ies available for review. For the same reason, no posttreatment SWI studies were available for review. All imaging was performed on a 1.5T unit; the depiction of venous congestion should theoretically be enhanced at a higher magnetic field, 

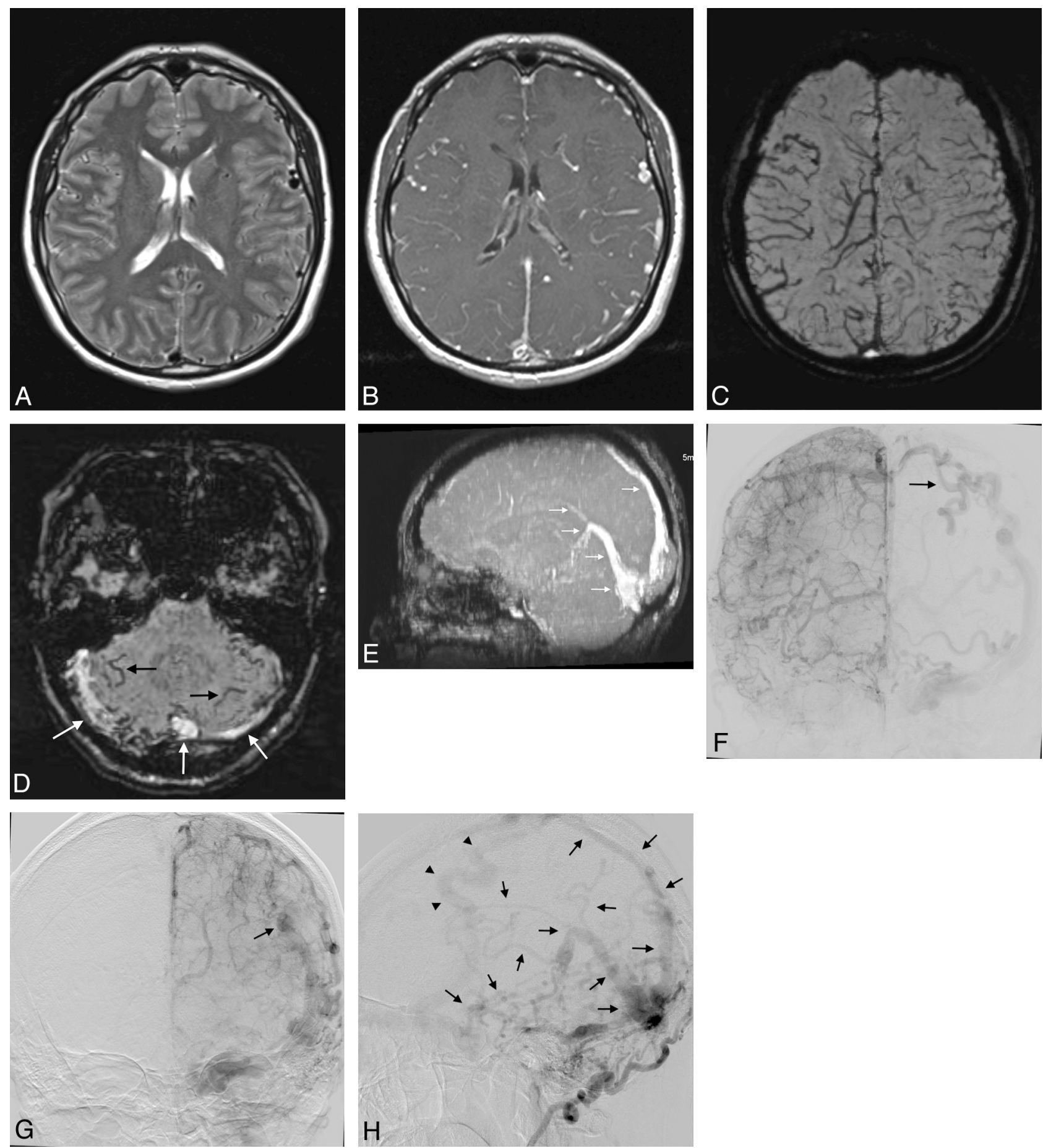

Fig 3. A 37-year-old woman who presented with tinnitus, harboring a Borden type II fistula affecting the right transverse sinus with retrograde flow in the superior sagittal sinus, torcula, straight sinus, and deep venous system as well as CVR (patient 5). There was also another fistulous connection draining in the superior sagittal sinus (not shown). $A$ and $B$, T2-weighted and T1-weighted postgadolinium administration images demonstrate dilated leptomeningeal and medullary vessels, more conspicuous in $B$. C, mIP of the SWI acquisition better demonstrates the extensive network of dilated leptomeningeal and medullary vessels. D, SWI image reveals the hypointense tortuous veins within the cerebellar hemispheres (black arrows) as well as hyperintensity within both transverse sinuses and the torcula (white arrows). E, MIP of the SWI images demonstrates hyperintensity in the torcula, straight sinus, vein of Galen, and inferior and superior sagittal sinuses (arrows). Note that the enlarged vein of Trolard (seen in $F, G$, and $H$ ) was not included in the volume of this MIP reformation but was visible on SWI images as a hyperintense vessel (not shown). Fand G, AP projections of the right and left internal carotid angiograms obtained in the venous phase. A severe PPP is seen in both hemispheres. The pattern is similar to the findings on SWI in B. The ectatic and tortuous vein of Trolard is seen on the left (arrow in $F$ and G). $H$, Lateral projection of a selective right occipital artery injection obtained in the late arterial phase. Extensive retrograde filling of the straight sinus, vein of Galen, and deep venous system as well as of the inferior and superior sagittal sinuses and of numerous cortical veins is demonstrated (arrows). The findings on SWI in $D$ and $E$ are similar but less extensive than on DSA. The enlarged left vein of Trolard is again demonstrated with CVR (arrowheads).

given the higher sensitivity to susceptibility artifacts. The identification of arteriovenous shunting was not altered in the series of Jagadeesan et al, ${ }^{10}$ which included patients evaluated both on $1.5 \mathrm{~T}$ and $3 \mathrm{~T}$ units. Finally, we acknowledge the fact that small DAVFs associated with mild PPP on SWI might be difficult to differentiate from normal veins when the diagnosis 
of DAVF is not known. The ability of SWI to distinguish normal venous structures and abnormal congested veins should ideally be evaluated in a larger study including a control group of patients not harboring a DAVF. In our limited experience, the prominent veins seen secondary to venous congestion in DAVFs were generally beyond the expected normal venous anatomy seen in our daily practice and, in fact, led to further investigations in these cases.

\section{Conclusions}

Although we present a small series of patients, our preliminary experience consolidates previous reports on the role of SWI in the diagnosis of DAVF. Not only can SWI depict the acute or chronic hemorrhagic complications of DAVF, but it may also help in the identification of the fistulous point. The exquisite sensitivity to susceptibility effects of SWI appears to provide unparalleled depiction of venous congestion, while CVR seems underestimated. In the presence of increased number, caliber, and tortuosity of hypointense leptomeningeal or medullary veins, a search for hyperintense venous structures on SWI images should be prompted, followed by diagnostic confirmation either by time-resolved CTA or MRA or by the use of conventional angiography. We believe that SWI has major potential clinical applications in DAVF diagnosis, prognostication, and posttreatment monitoring. Its role in the diagnostic armamentarium of DAVFs needs to be further assessed in larger studies.

\section{References}

1. Wilson M, Enevoldson P, Menezes B. Intracranial dural arterio-venous fistula. Pract Neurol 2008;8:362-69

2. Dehdashti AR, Matouk CC, Terbrugge $\mathrm{K}$, et al. Clues to dural arteriovenous fistulas in patients with progressive dementia. Can J Neurol Sci 2010;37:532-34

3. Willinsky R, Goyal M, terBrugge $\mathrm{K}$, et al. Tortuous, engorged pial veins in intracranial dural arteriovenous fistulas: correlations with presentation, location, and MR findings in 122 patients. AJNR Am J Neuroradiol 1999;20:1031-36

4. Cognard C, Gobin YP, Pierot L, et al. Cerebral dural arteriovenous fistulas: clinical and angiographic correlation with a revised classification of venous drainage. Radiology 1995; 194:671-80

5. Davies MA, TerBrugge K, Willinsky R, et al. The validity of classification for the clinical presentation of intracranial dural arteriovenous fistulas. J Neurosurg 1996;85:830-37

6. Borden JA, Wu JK, Shucart WA. A proposed classification for spinal and cranial dural arteriovenous fistulous malformations and implications for treatment. J Neurosurg 1995;82:166-79

7. Saini J, Thomas B, Bodhey NK, et al. Susceptibility-weighted imaging in cranial dural arteriovenous fistulas. AJNR Am J Neuroradiol 2009;30:E6

8. Noguchi K, Kuwayama N, Kubo M, et al. Intracranial dural arteriovenous fistula with retrograde cortical venous drainage: use of susceptibilityweighted imaging in combination with dynamic susceptibility contrast imaging. AJNR Am J Neuroradiol 2010;31:1903-10

9. Gasparetto EL, Pires CE, Domingues RC. Susceptibility-weighted MR phase imaging can demonstrate retrograde leptomeningeal venous drainage in patients with dural arteriovenous fistula. AJNR Am J Neuroradiol 2011;32:E54. Epub 2011 Feb 17
10. Jagadeesan BD, Delgado Almandoz JE, Moran CJ, et al. Accuracy of susceptibility-weighted imaging for the detection of arteriovenous shunting in vascular malformations of the brain. Stroke 2011;42:87-92

11. Tsui YK, Tsai FY, Hasso AN, et al. Susceptibility-weighted imaging for differential diagnosis of cerebral vascular pathology: a pictorial review. J Neurol Sci 2009;287:7-16

12. Haacke EM, Mittal S, Wu Z, et al. Susceptibility-weighted imaging: technical aspects and clinical applications, part 1. AJNR Am J Neuroradiol 2009;30:19-30

13. Kwon BJ, Han MH, Kang HS, et al. MR imaging findings of intracranial dural arteriovenous fistulas: relations with venous drainage patterns. AJNR Am J Neuroradiol 2005;26:2500-07

14. Willems PW, Brouwer PA, Barfett JJ, et al. Detection and classification of cranial dural arteriovenous fistulas using 4D-CT angiography: initial experience. AJNR Am J Neuroradiol 2011;32:49-53

15. Farb RI, Agid R, Willinsky RA, et al. Cranial dural arteriovenous fistula: diagnosis and classification with time-resolved MR angiography at 3T. AJNR Am J Neuroradiol 2009;30:1546-51

16. Nishimura S, Hirai T, Sasao A, et al. Evaluation of dural arteriovenous fistulas with $4 \mathrm{D}$ contrast-enhanced MR angiography at 3T. AJNR Am J Neuroradiol 2010;31:80-85

17. Hiu T, Kitagawa N, Morikawa M, et al. Efficacy of DynaCT digital angiography in the detection of the fistulous point of dural arteriovenous fistulas. AJNR Am J Neuroradiol 2009;30:487-91

18. Brouwer PA, Bosman T, van Walderveen MA, et al. Dynamic 320-section CT angiography in cranial arteriovenous shunting lesions. AJNR Am J Neuroradiol 2010;31:767-70

19. Lee CW, Huang A, Wang YH, et al. Intracranial dural arteriovenous fistulas: diagnosis and evaluation with 64-detector row CT angiography. Radiology 2010;256:219-28

20. Hu J, Yu Y, Juhasz C, et al. MR susceptibility weighted imaging (SWI) complements conventional contrast enhanced T1 weighted MRI in characterizing brain abnormalities of Sturge-Weber Syndrome. J Magn Reson Imaging 2008;28:300-07

21. Iwama T, Hashimoto N, Takagi Y, et al. Hemodynamic and metabolic disturbances in patients with intracranial dural arteriovenous fistulas: positron emission tomography evaluation before and after treatment. J Neurosurg 1997;86:806-11

22. Kuroda S, Furukawa K, Shiga T, et al. Pretreatment and posttreatment evaluation of hemodynamic and metabolic parameters in intracranial dural arteriovenous fistulae with cortical venous reflux. Neurosurgery 54:585-91, 2004, discussion 591-82

23. Metoki T, Mugikura S, Higano S, et al. Subcortical calcification on CT in dural arteriovenous fistula with cortical venous reflux. AJNR Am J Neuroradiol 2006;27:1076-78

24. Yang MS, Chen CC, Cheng YY, et al. Unilateral subcortical calcification: a manifestation of dural arteriovenous fistula. AJNR Am J Neuroradiol 2005;26:1149-51

25. Mittal S, Wu Z, Neelavalli J, et al. Susceptibility-weighted imaging: technical aspects and clinical applications, part 2. AJNR Am J Neuroradiol 2009;30:232-52

26. Robinson RJ, Bhuta S. Susceptibility-weighted imaging of the brain: current utility and potential applications. J Neuroimaging 2011 Jan 31. [Epub ahead of print]

27. Kesavadas C, Santhosh K, Thomas B. Susceptibility weighted imaging in cerebral hypoperfusion: can we predict increased oxygen extraction fraction? Neuroradiology 2010;52:1047-54

28. Karaarslan E, Ulus S, Kurtuncu M. Susceptibility-weighted imaging in migraine with aura. AJNR Am J Neuroradiol 2011;32:E5-7

29. Altinok D, Agarwal A, Ascadi G, et al. Pediatric hemiplegic migraine: susceptibility weighted and MR perfusion imaging abnormality. Pediatr Radiol 2010;40:1958-61

30. Santhosh K, Kesavadas C, Thomas B, et al. Susceptibility weighted imaging: a new tool in magnetic resonance imaging of stroke. Clin Radiol 2009;64:74-83

31. Leach JL, Strub WM, Gaskill-Shipley MF. Cerebral venous thrombus signal intensity and susceptibility effects on gradient recalled-echo MR imaging. AJNR Am J Neuroradiol 2007;28:940-45 